\title{
ACUIDADE AUDITIVA EM ALTAS FREQUÊNCIAS E CONTAMINAÇÃO MICROBIANA DOS PLUGS DE OUVIDO EM ESTUDANTES UNIVERSITÁRIOS
}

LIMA, Fabiana Regina Sabion Giacheto de ${ }^{1}$ KOZUSNY-ANDREANI, Dora Inés ${ }^{2}$

\begin{abstract}
RESUMO: Se diversas espécies, sendo Candida albicans, Staphylococcus aureus e Micrococcus spp isolados Com a popularização dos dispositivos sonoros portáteis individuais, entre os adolescentes, aliados a alta tecnologia, o som intenso pode lesar estruturas da orelha e consequentemente acarretar em uma perda auditiva. Indivíduos expostos ao ruído podem apresentar também disfunções vestibulares. Objetivou-se no presente estudo analisar o impacto do ruído no sistema auditivo e vestibular em adolescentes usuários de dispositivos sonoros portáteis individuais, assim como a microbiota dos plugs de ouvido e relacionar com os microrganismos das orelhas. Para avaliação da função coclear foram realizados os testes de audiometria tonal e vocal, audiometria de alta frequência e imitanciometria. Os sintomas otoneurológicos foram avaliados com o Dizziness Handicap Inventory. A contaminação microbiana foi realizada pela técnica "swab test" dos plugs e de orelhasdos estudantes universitarios. Os resultados foram analisados estatisticamente para verificar a significância e a correlação dos achados. Alterações nos limiares auditivos não foram detectados na avaliação audiológica convencional na maioria $(91,66 \%)$ dos estudantes universitários. Ao qualificar, quantificar e correlacionar as colônias de bactérias e fungos encontrados nos plugs de inserção e na orelha direita e orelha esquerda, verificoucom maior frequência.
\end{abstract}

Palavras-chave: Ruído ocupacional. Cóclea. Sistema vestibular. Micro-organismos.

\section{AUDITORY ACUITY AT HIGH FREQUENCIES AND MICROBIAL CONTAMINATION OF PLUGS OF HEARING IN UNIVERSITY STUDENTS}

\begin{abstract}
SUMMARY: With the popularization of individual portable sound devices, among teenagers, allied to high technology, the intense sound can damage the structures of the ear and consequently lead to a hearing loss. Individuals exposed to noise may also have vestibular dysfunctions. In addition to these changes, these can acquire hearing losses arising from the colony of bacteria in the plugs that are used. The objective of this study was to analyze the impact of noise in the auditory and vestibular system in adolescents using individual portable sound devices, as well as the microbiota of the ear plugs and to relate to the microorganisms of the ears. For the evaluation of the cochlear function, the audiometry and tonal and vocal audiometry, high frequency audiometry and immitance tests were performed. Otoneurological symptoms were evaluated with the Dizziness Handicap Inventory. Microbial contamination was performed by the "swab test" technique of plugs and ear in university students. The results were statistically analyzed to verify the significance and correlation of the findings. Changes in auditory thresholds were not detected in the conventional audiological evaluation in the majority (91.66\%) of university students. In order to qualify, quantify and correlate colonies of bacteria and fungi found in the insertion plugs and in the right ear and left ear, several species were found, being Candida albicans, Staphylococcus aureus and Micrococcus spp more frequently isolated.
\end{abstract}

Keywords: Occupational noise. Cochlea. Vestibular System. Microorganisms.

\section{INTRODUÇÃO}

A perda auditiva induzida por níveis de pressão sonora elevada (PAINPSE) era, tradicionalmente, uma doença de adultos que trabalhavam em ocupações ruidosas. No entanto, desde o advento da música

\footnotetext{
${ }^{1}$ Fonoudióloga, Mestre em Ciências Ambientais - Fundação Educacional de Fernandópolis

${ }^{2}$ Professora/Pesquisadora. Mestrado em Ciências Ambientais. Campus Fernandópolis - Universidade Brasil
} 
amplificada e a crescente popularização dos dispositivos sonoros portáteis individuais, entre os adolescentes, este se tornou um hábito constante entre os mesmos (SANT'ANA, 2012)

A qualidade dos aparelhos e plugs de ouvido também evoluíram, permitindo maiores níveis de volume com melhor correção de eventuais distorções, o que possibilita aos usuários ouvir música em volumes elevados sem incomodar as pessoas ao redor. Atualmente, é comum encontra crianças e adolescentes fazendo o uso de fones auriculares em diversas situações. Os fones do tipo "plug" são os preferidos, uma vez que geram uma maior pressão sonora sobre a membrana timpânica. Paralelamente, tem se verificado uma maior preocupação da comunidade científica e da sociedade com os possíveis efeitos deletérios do uso intensivo destes dispositivos sonoros. (HODGETTS; IEGER; SZARKO, 2007)

A exposição contínua a níveis de pressão sonora elevados, profissional ou não, pode resultar em lesões nas células ciliadas e no nervo auditivo através de variados mecanismos. Sendo esta, a segunda causa mais comum da deficiência auditiva (DOBIE, 1998).

O método empregado para o diagnóstico e acompanhamento da PAINPSE é a audiometria tonal liminar, enquanto a audiometria de altas frequências é útil no diagnóstico precoce (LOPES et al., 2009). A audiometria tonal limiar de altas frequências (AT-AF), possui o objetivo de avaliar frequências entre 9.000 e $18.000 \mathrm{~Hz}$ (LOPES; MELO; SANTOS, 2012).

Além de alterações auditivas, indivíduos expostos ao ruído podem apresentar disfunções vestibulares. Ao se correlacionar o ruído com sintomas vestibulares pode ser verificado durante a exposição ao ruído ou mesmo após, presença de alterações vestibulares, descritas como vertigens, que podem ou não ser acompanhadas de náuseas, vômitos, suores frios e dificuldades no equilíbrio e na marcha (TEIXEIRA; KÖRBES; ROSSI, 2011).

A intervenção fonoaudiológica junto à saúde auditiva e vestibular do adolescente usuário de dispositivos sonoros portáteis individuais estéreos tem como objetivo prevenir ou minimizar as consequências biológicas e sociais da PAINPSE. O trabalho atua no controle do desencadeamento ou agravamento de lesões auditivas e vestibulares, desenvolvendo medidas educacionais de promoção e prevenção a saúde auditiva, fornecendo melhorias na qualidade de vida (LOPES; MELO; SANTOS, 2012).

A literatura demonstra que somente a audiometria tonal convencional pode não ser eficaz na prevenção e identificação precoce da PAINPSE. Quanto ao comprometimento do sistema vestibular, as pesquisas são escassas, mesmo porque lesões no labirinto podem não manifestar sintomas devido à capacidade de sistema em se adaptar ao dano provocado pelos sons de alta intensidade (LAITINEN, 2005).

Além das alterações auditivas e vestibulares decorrentes do uso dos dispositivos sonoros portáteis individuais, estes podem adquirir outras alterações decorrentes da contaminação dos plugs, por bactérias e fungos potencialmente patogênicos, devido à falta de higienização adequada dos mesmos. O emprego destes equipamentos contaminados pode provocar infecção dos ouvidos. Segundo Wasihun, Zemene (2015), a etiologia e a frequência da infecção do ouvido são diferentes e estão de acordo com o ambiente. No entanto, os micro-organismos mais frequentes isolados de casos de otite são: Pseudomonas aeruginosa, Staphylococcus aureus, Proteus mirabilis, Klebsiella pneumoniae, Escherichia coli, Streptococcus pneumoniae, Streptococcus pyogenes e Candida albicans (MULUYE et al. 2013; SEID et al. 2013; HAILU et al., 2016; ILECHUKWU et al., 2017; HAILEGIYORGIS, SARHIE, WORKIE, 2018). Dessa forma, objetivou-se neste estudo avaliar o impacto do ruído no sistema auditivo e vestibular em estudantes universitários usuários de dispositivos sonoros portáteis individuais (plugs), assim como a microbiota dos plugs de ouvido e relacionar com os micro-organismos do conduto auditivo externo. 


\section{MATERIAL E MÉTODO}

A pesquisa foi desenvolvida após aprovação do Comitê de Ética em Pesquisa do Sistema Nacional de ética e Pesquisa (SISNEP), com CAAE: 60460016.2.0000.5494. Os participantes do estudo, após serem informados sobre todos os detalhes do trabalho, manifestaram o seu consentimento por escrito, assinando o Termo de Consentimento Livre e Esclarecido.

O estudo foi realizado na Clínica de Audiologia das Faculdades Integradas de Fernandópolis (FIFE), Fernandópolis- SP.

Para compor a casuística do estudo, foram selecionados 24 sujeitos de ambos os gêneros, na faixa etária entre 17 e 24 anos de idade.

Os sujeitos selecionados foram adolescentes universitários. Todos foram submetidos à avaliação audiológica de acordo com os critérios estabelecidos pelo Ministério da Saúde no seu Programa de Saúde Auditiva.

Os critérios de inclusão foram: ser estudante universitário, aceitar participar da pesquisa, estar dentro da faixa etária proposta e ser usuário de dispositivos sonoros portáteis individuais (plugs).

Os critérios de exclusão foram: apresentar doenças como caxumba, hipertensão arterial, diabetes, meningite, HIV, sífilis, lúpus eritomatoso sistemico, artite reumatoide, arteriosclerose e hipercolesterolemia que possam comprometer a acuidade auditiva, e ou deficiência auditiva pré-existente; além da não aceitação dos sujeitos na participação.

Inicialmente foi realizada a medição do ruído dos dispositivos sonoros portáteis individuais dos adolescentes, quando ligados por meio do equipamento decibelímetro (marca IMPAC modelo 8921).

A pesquisa da função coclear foi realizada uma entrevista específica com o intuito de investigar as características individuais, tais como idade, hábitos de lazer ruidosos, exposição a produtos químicos, além das condições de saúde e outras doenças que possam potencializar os efeitos dos riscos ambientais.

Após a entrevista foi realizada a inspeção do meato acústico externo. Com o uso de otoscópio, com a finalidade de se avaliar a membrana timpânica e o meato acústico externo.

Para verificação da acuidade auditiva foi realizada a audiometria tonal liminar convencional (250 a $8.000 \mathrm{~Hz}$ ); audiometria tonal liminar de altas frequências $(9.000$ e $12.000 \mathrm{~Hz})$, e logoaudiometria: foram realizadas com o audiômetro GSI-61, marca GrassonStadler. Foi utilizado o tom warble para pesquisas dos limiares tonais, os quais foram apresentados por meio dos fones auriculares HDA 200. Para a pesquisa dos limiares tonais, foi utilizada a técnica descendente. A classificação utilizada para o tipo de perda foi de acordo com Silman e Silverman (1997) e quanto ao grau da perda BIAP (1996)

Com o intuito de se avaliar a funcionalidade da orelha média foi realizada a pesquisa da medida da imitância acústica e a pesquisa dos reflexos ipsilaterais e contralaterais do músculo estapédio. Para tal, foi utilizado com o equipamento AZ-7R. Estas foram classificadas de acordo com a proposta de JERGER (1970).

O Protocolo de Dizziness Handicap Inventory (DHI) foi utilizado para quantificar o sintoma específico da tontura, que pode estar presente nos adolescentes.

Para avaliação da microbiota dos plugs e do conduto auditivo externo, foram realizadas amostragens por meio da técnica "swab test". Para tal finalidade um swab estéril foi friccionado na superfície do plug, em zig zag e sempre em um único sentido, as amostras colhidas foram depositadas em meio de transporte (Stuart), identificadas e conservadas em caixa isotérmicas a $4^{\circ} \mathrm{C}$.

Para a detecção de micro-organismos patogênicos do conduto auditivo externo, todas as amostras foram coletadas por técnica microbiológica padrão (HAILU et al., 2016), utilizou-se swab e transportadas em meio Stuart para analises microbiológicas. 
Para cultivo as amostras foram submetidas a diluições seriadas em solução salina $(\mathrm{NaCl}, 0,5 \%)$ posteriormente cultivado em meios agarizados BHI, triptecaseina soja (TSA), MacConkey e Sabouraud (OXOID ${ }^{\circledR}$ ), sendo incubadas a $37^{\circ} \mathrm{C}$ por $24-48$ horas, quando as colônias foram avaliadas pelas características morfológicas.

As colônias foram submetidas à coloração de Gram e observadas em microscópio de luz, utilizando a objetiva de 100x, que proporciona um aumento de 1000x. Uma vez confirmada à característica morfológica cada colônia foi cultivada isoladamente em meios agarizados para posteriormente ser avaliadas por meio de provas bioquímicas para a sua identificação. Colônias típicas de estafilicocos foram submetidas as provas bioquímicas da catalase, coagulase, hemólise, e oxidase (WINN et al., 2008), enquanto que bactérias Gram-negativas foram avaliadas pelo Sistema API20E (Analytical Profile Index, BioMérieux). Para identificação das leveduras foram adotas as recomendações descritas por Winn et al (2008).

Os dados obtidos foram analisados por meio de estatística descritiva e métodos estatísticos inferenciais analisando-se questões de probabilidade de uma população com base nos dados da amostra. Variáveis discretas foram expressas como porcentagens. O teste do qui-quadrado foi empregado e o valor de $\mathrm{p}<0,05$ foi considerado estatisticamente significante. Os softwares estatísticos utilizados para a análise foi o Software SPSS Statistics atreladas às funcionalidades da ferramenta Excel (versão 2.016)

\section{RESULTADO}

Para pesquisa da função coclear participaram 24 adolescentes universitários com idade entre 17 a 24 anos. Sendo 9 do gênero masculino e 15 do gênero feminino. Por meio da entrevista específica cujo intuito era investigar as características individuais, tais como hábitos de lazer ruidosos, exposição a produtos químicos, além das condições de saúde e outras doenças que possam potencializar os efeitos dos riscos ambientais. Nesta entrevista 20\% dos adolescentes referiram hábitos de lazer ruidoso com o uso de dispositivos sonoros portáteis individuais com plugs de inserção, para música em volume elevado por mais de duas horas por dia. No entanto, nenhum referiu exposição a produtos químicos, além das condições de saúde e outras doenças que poderiam potencializar os efeitos dos riscos ambientais.

Para verificação da acuidade auditiva foi realizada a audiometria tonal liminar convencional (250 a $8.000 \mathrm{~Hz}$ ); audiometria tonal liminar de altas frequências $(9.000$ e $12.000 \mathrm{~Hz})$, e logoaudiometria. Os resultados apresentados no gráfico 1 evidenciam que 8,34\% (n=2) dos universitários apresentaram alterações auditivas para altas frequências na orelha direita e orelha esquerda, enquanto que em 91,66\% $(n=22)$, os limiares tonais eram normais para altas frequências. Em relação à audiometria tonal liminar convencional (250 a $8.000 \mathrm{~Hz})$, nenhum universitário apresentou alteração auditiva.

Grafico 1: Resultados da audiometria das orelhas direita e esquerda de estudantes universitários, usuários de dispositivos sonoros portáteis individuais no município de Fernandópolis- SP.

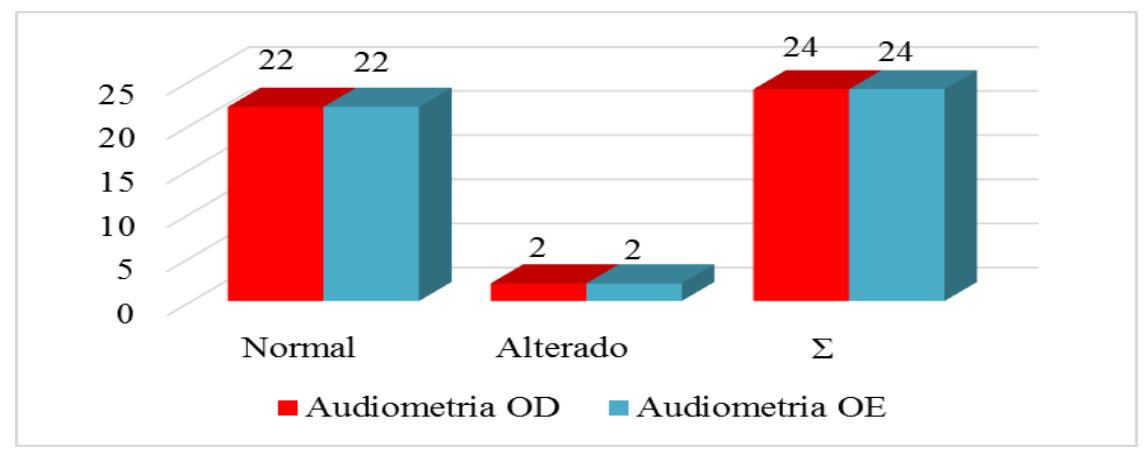


Com o intuito de avaliar a funcionalidade da orelha média foi realizada a pesquisa da medida da imitância acústica e a pesquisa dos reflexos ipsilaterais e contralaterais do músculo estapédio. Nesta análise constatou-se que 92,66\% ( $\mathrm{n}=22)$ dos universitários apresentaram curva timpanométria do tipo A e 8,34\% $(n=2)$ curva timpanométria do tipo Ad, na orelha direita. Na orelha esquerda, $87,5 \%(n=21)$ apresentaram curva timpanométria do tipo A e 13,5\% (n=3) do tipo Ad (Gráfico 2).

Gráfico 2: Curva timpanométria quanto ao tipo- orelha direita e orelha esquerda de estudantes universitários, usuários de dispositivos sonoros portáteis individuais no município de Fernandópolis- SP

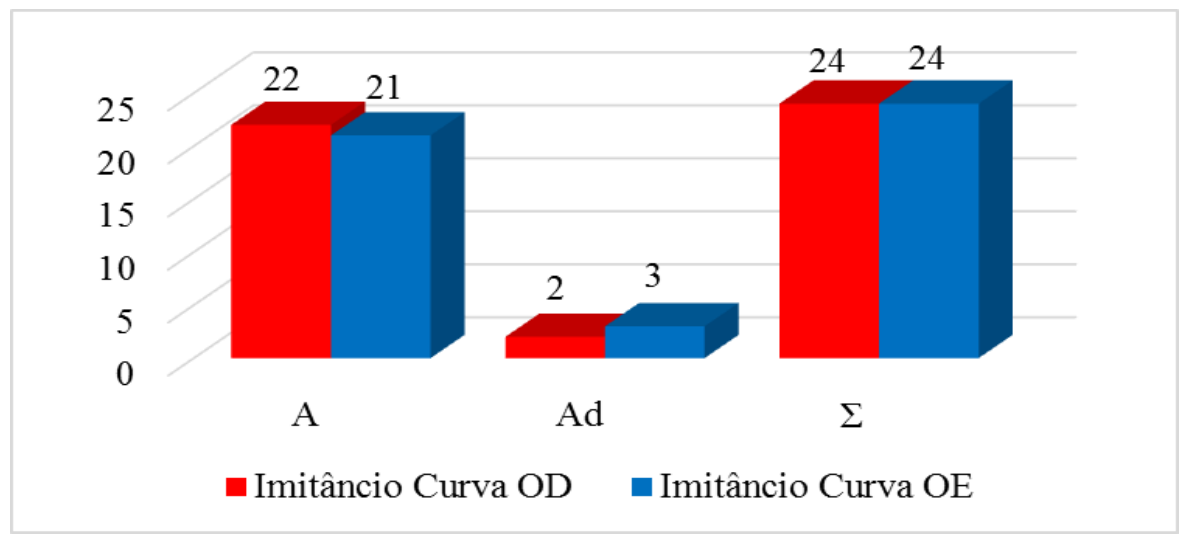

Quanto a pesquisa dos reflexos ipsilaterais e contralaterais do músculo estapédio, contatou-se que $100 \%$ dos estudantes apresentaram presença dos reflexos ipsilaterais e contralaterais para a orelha direita e esquerda.

O Protocolo de Dizziness Handicap Inventory (DHI) foi utilizado para quantificar o sintoma específico da tontura, constatou-se que $83,34 \%(\mathrm{n}=20)$ dos estudantes não apresentaram queixas labirínticas e apenas $16,66 \%(n=4)$ apresentaram tontura (Gráfico 3).

Gráfico 3: Alterações labirínticas de estudantes universitários, usuários de dispositivos sonoros portáteis individuais no município de Fernandópolis- SP.

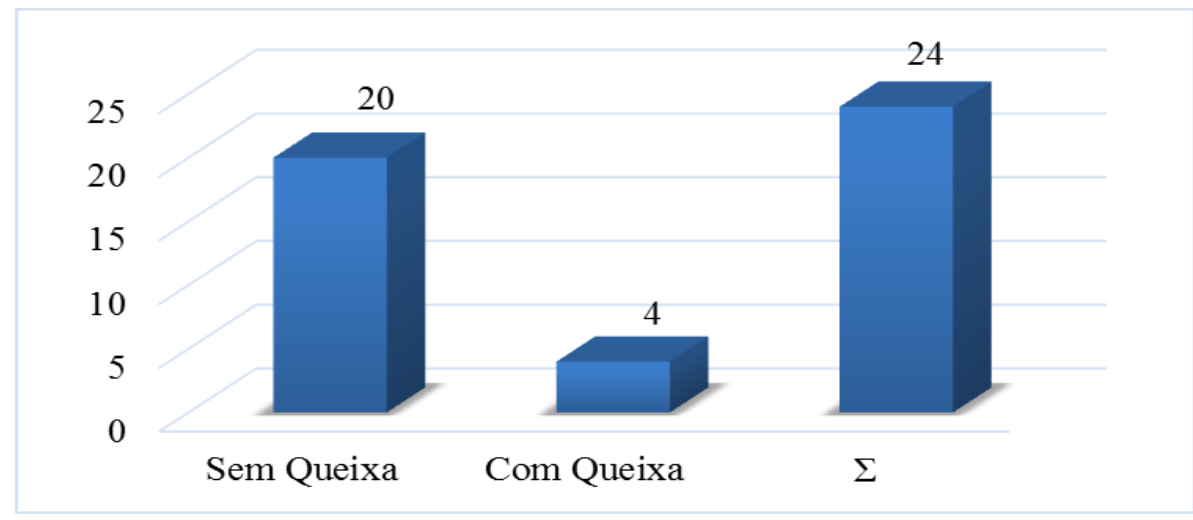

Na tabela 1 são apresentados os resultados referentes ao perfil microbiológico de plugs e de orelhas direita e esquerda de estudantes universitários, usuários de dispositivos sonoros portáteis individuais. Verificou-se prevalência de Candida albicans nas amostras de plugs e de orelhas esquerda e direita, no entanto Staphylococcus aureus e Micrococcus spp foram frequentemente isolados (Tabela 2). 
Tabela 1: Perfil microbiológico de plugs e de orelhas direita e esquerda de estudantes universitários, usuários de dispositivos sonoros portáteis individuais no município de Fernandópolis- SP

\begin{tabular}{|c|c|c|c|c|}
\hline \multirow[t]{2}{*}{ Participante } & \multicolumn{4}{|c|}{ Microbiota } \\
\hline & Orelha esquerda & Orelha direita & Plug direito & Plug esquerdo \\
\hline 1 & $\begin{array}{l}\text { C. } \text { albicans }^{1} \\
\text { S. aureus }\end{array}$ & $\begin{array}{c}\text { C. albicans } \\
\text { S. aureus }\end{array}$ & C. albicans & $\begin{array}{c}\text { C. albicans } \\
\text { S. aureus }\end{array}$ \\
\hline 2 & C. albicans & $\begin{array}{l}\text { C. albicans } \\
\text { S. aureus }\end{array}$ & C. albicans & C. albicans \\
\hline 3 & C. albicans & C. albicans & C. albicans & C; albicans \\
\hline 4 & C. albicans & C. albicans & C. albicans & C. albicans \\
\hline 6 & C. albicans & P. aeruginosa ${ }^{3}$ & P. aeruginosa & P. aeruginosa \\
\hline 7 & C. albicans & C. albicans & C. albicans & C. albicans \\
\hline 8 & $\begin{array}{c}\text { C. albicans } \\
\text { P. aerugimosa } \\
\text { Micrococcus spp }\end{array}$ & $\begin{array}{c}\text { S. aureus } \\
\text { Micrococcus spp }\end{array}$ & $\begin{array}{l}\text { S. aureus } \\
\text { Micrococcus spp }\end{array}$ & $\begin{array}{l}\text { P. aerugimosa } \\
\text { Micrococcus spp }\end{array}$ \\
\hline 9 & C. albicans & C. albicans & C. albicans & C. albicans \\
\hline 10 & C. albicans & C. albicans & C. albicans & C. albicans \\
\hline 11 & C. albicans & C. albicans & C. albicans & C. albicans \\
\hline 12 & C. albicans & C. albicans & C. albicans & C. albicans \\
\hline 13 & $\begin{array}{c}\text { C. albicans } \\
\text { S. epidermidis }{ }^{4} \\
\text { Streptococcus spp } \\
\text { Pseudomonas spp }\end{array}$ & C. albicans & Candida albicans & $\begin{array}{c}\text { C. albicans } \\
\text { S. epidermidis }\end{array}$ \\
\hline 14 & $\begin{array}{c}\text { S. aureus } \\
\text { Micrococcus spp }\end{array}$ & $\begin{array}{c}\text { S. aureus } \\
\text { Micrococcus spp }\end{array}$ & $\begin{array}{c}\text { S. aureus } \\
\text { Micrococcus spp }\end{array}$ & $\begin{array}{l}\text { P. aerugimosa } \\
\text { Micrococcus spp }\end{array}$ \\
\hline 15 & C. albicans & C. albicans & Candida albicans & C. albicans \\
\hline 16 & C. albicans & $\begin{array}{l}\text { C. albicans } \\
\text { S. aureus }\end{array}$ & $\begin{array}{l}\text { C. albicans } \\
\text { S. aureus }\end{array}$ & C, albicans \\
\hline 17 & $\begin{array}{l}\text { C. albicans } \\
\text { S. aureus }\end{array}$ & $\begin{array}{l}\text { C. albicans } \\
\text { S. aureus }\end{array}$ & $\begin{array}{l}\text { C. albicans } \\
\text { S. aureus } \\
\text { Bacillus spp }\end{array}$ & $\begin{array}{l}\text { C. albicans } \\
\text { S. aureus }\end{array}$ \\
\hline 18 & C. albicans & C. albicans & C. albicans & C. albicans \\
\hline 19 & C. albicans & C. albicans & C. albicans & C. albicans \\
\hline 20 & $\begin{array}{c}\text { C. albicans } \\
\text { S. aureus } \\
\text { Micrococcus spp }\end{array}$ & $\begin{array}{c}\text { C. albicans } \\
\text { S. aureus } \\
\text { Micrococcus spp }\end{array}$ & $\begin{array}{l}\text { C. albicans } \\
\text { S. aureus }\end{array}$ & $\begin{array}{c}\text { C. albicans } \\
\text { Micrococcus spp }\end{array}$ \\
\hline 21 & $\begin{array}{l}\text { C. albicans } \\
\text { S. aureus }\end{array}$ & $\begin{array}{l}\text { C. albicans } \\
\text { S. aureus }\end{array}$ & $\begin{array}{l}\text { C. albicans } \\
\text { S. aureus }\end{array}$ & $\begin{array}{c}\text { C. albicans } \\
\text { Micrococcus spp }\end{array}$ \\
\hline 21 & C. albicans & C. albicans & C. albicans & C. albicans \\
\hline 23 & C. albicans & C. albicans & C. albicans & C. albicans \\
\hline 24 & $\begin{array}{l}\text { C. albicans } \\
\text { S. aureus }\end{array}$ & $\begin{array}{l}\text { C. albicans } \\
\text { S. aureus }\end{array}$ & $\begin{array}{l}\text { C. albicans } \\
\text { S. aureus }\end{array}$ & $\begin{array}{l}\text { C. albicans } \\
\text { S. aureus }\end{array}$ \\
\hline
\end{tabular}


Pela análise estatística descritiva individual das variáveis usuários e total de microrganismos isolados, foi detectada a ocorrência de 144 micro-organismos. Assim, verificou-se que Candida albicans apresentou 89 ocorrências (61,8\%) dos casos, Staphylococcus aureus 28 (19,44\%) e Micrococcus spp 13 (9,03\%), considerados mais frequentes quando comparados com os demais isolados (Tabela 2). Uma outra característica observada foi a presença dos mesmos micro-organismos nas orelhas e nos plugs, na maioria da amostras avaliadas (Tabela 1).

Tabela 2: Frequência de isolamento dos micro-organismos plugs e de orelhas direita e esquerda de estudantes universitários, usuários de dispositivos sonoros portáteis individuais no município de Fernandópolis- SP.

\begin{tabular}{|c|c|c|}
\hline \multicolumn{3}{|c|}{ Perfil Microbiológico das Amostras } \\
\hline Micro-organismos & Registros (N) & Proporção (\%) \\
\hline Candida albicans & 89 & 61,81 \\
\hline Staphylococcus aureus & 28 & 19,44 \\
\hline Staphylococcus epidermidis & 2 & 1,39 \\
\hline Bacillus spp & 4 & 2,78 \\
\hline Micrococcus spp & 13 & 9,03 \\
\hline Pseudomonas aeruginsa & 6 & 4,17 \\
\hline Pseudomonas spp & 1 & 0,69 \\
\hline Streptococcus spp & 1 & 0,69 \\
\hline Total de isolados & 144 & 100,00 \\
\hline Desvio Padrão $^{1}$ & \multicolumn{2}{|c|}{30,09} \\
\hline Erro Padrão ${ }^{2}$ & \multicolumn{2}{|c|}{8,04} \\
\hline
\end{tabular}

\section{DISCUSSÃO}

O advento da música amplificada e a crescente popularização dos dispositivos sonoros portáteis individuais, entre os adolescentes, e a disponibilidade de marcas e modelos, além da alta tecnologia possibilitou o acesso a essa tecnologia. Entretanto, se esta tecnologia for utilizada de forma inapropriada, isto é, uso abusivo da duração, intensidade e/ou frequência da exposição, esta poderá acarretar em impacto adverso na função auditiva (KEPPLER et al., 2010; KNOBEL, LIMA, 2011; SANT'ANA, 2012; BARCELOS, DAZZI, 2014; SILVESTRE et al., 2016; MONDELLI et al., 2016). Além de sintomas auditivos os usuários podem apresentar sintomas extra auditivos, tais como dificuldade de concentração e atenção, memória, nervosismo e fadiga excessiva. Além disso, estudos mostram que a exposição ao ruído afeta o sistema nervoso simpático e endócrino, resultando em respostas fisiológicas como aumento da frequência cardíaca, aumento da pressão arterial e vasoconstrição (OLIVEIRA et al., 2015).

Kleppler et al. (2010), ao avaliar os feitos auditivos de curto prazo em in divíduos usuários de MP3 player, foi verificado que o grupo de exposição a ruídos levasse a mudanças significativas nos limiares auditivos e nas amplitudes das emissões otoacústicas, quando comparados com o grupo controle sem exposição. Os autores salientam ainda, que as alterações temporárias na sensibilidade auditiva medidas 
por audiometria e emissões otoacústicas indicam os potenciais efeitos prejudiciais da audição de um MP3 player. Na presente pesquisa em relação à audiometria tonal liminar convencional $(250 \mathrm{a} 8.000 \mathrm{~Hz})$, nenhum universitário apresentou alteração auditiva.

Segundo Araújo (2002) a audiometria de altas frequências é um instrumento de detecção precoce, de perda auditiva e de monitoramento da audição. Essa avaliação pode ser utilizada como medida profilática para a preservação da audição ou para evitar a possível progressão de uma perda auditiva já existente e a sensibilidade auditiva em altas frequências é importante para a compreensão de fala (LOPES et al., 2007).

Lopes et al. (2007) apontam a importância da mensuração adequada dos limiares de audibilidade de altas frequências para fins de deteç̧ão precoce de patologias cocleares e, consequentemente, a utilização de medidas profiláticas para a preservação da audição ou para evitar possível progressão de uma perda auditiva já existente. A audiometria de altas freqüências avalia sons acima de $8000 \mathrm{~Hz}$. É acima dessa frequência que a maioria das perdas auditivas neurossensoriais se inicia, dificilmente sendo percebidas pelos pacientes por estarem localizadas na base do ducto coclear, pouco influenciando a compreensão da fala. No presente estudo nenhum dos universitários apresentou alteração na audiometria tonal liminar convencional e na audiometria tonal em altas frequências foram encontradas alterações auditivas em $8,34 \%$ dos adolescentes avaliados.

Como sintomas auditivos os indivíduos podem apresentar queixa como o zumbido, cefaleia, plenitude auricular, tontura, distúrbios gástricos, da visão, do sono e do humor. Portanto, os adolescentes devem ser conscientizados sobre o ruído bem como sobre as consequências danosas que representam para a sua saúde (LOPES, MELO, SANTOS, 2012; GONÇALVES, DIAS, 2014).

A perda auditiva induzida por música, tem as mesmas características da perda auditiva induzida por ruído. A única diferença entre ambas encontra-se no conceito, uma vez que o ruído é definido como um som indesejado, e a música como algo prazeroso. O risco de perda auditiva não existe somente após longa exposição à música amplificada, pois curtas exposições a níveis sonoros excessivamente elevados também pode causar perda auditiva e zumbido (KEPPLER et al., 2010; KNOBEL, LIMA, 2015; SILVESTRE et al., 2016, OLIVEIRA et al. 2017).

Além de implicações auditivas, os usuários podem apresentar alterações extra auditivas, tais como alterações psicossociais, estresse, dificuldade de sono, depressão; além de transtornos neurológicos, vestibulares, digestivos, cardiovasculares e hormonais, tonturas, intolerância a sons intensos, otalgia e principalmente, o zumbido. A sensação de plenitude auricular e dor no conduto devido ao uso do fone de inserção também foram relatadas em vários estudos (MONDELLI et al., 2016). Durante a entrevista foi referenciado o zumbido, dor no conduto e otalgia quanto expostos por longos períodos de tempo. No entanto, os estudantes universitários avaliados referiram ouvir música por prazer e esta música muitas vezes é ouvida em volume elevado, o que acarreta em danos para o sistema auditivo. De acordo com Oliveira et al. (1017), esse problema se agrava quando os fones não se encaixam perfeitamente nos condutos auditivos, permitindo assim a entrada de sons externos, induzindo ao usuário a aumentar a intensidade de seus equipamentos, para mascarar os sons externos.

De acordo com Mazlan et al. (2002) o uso fones de ouvido ou tampões de ouvido pode ser considerado como possível fator predisponente para infecção do canal auditivo externo, já que seu uso pode aumentar a temperatura e a umidade do canal, criar o potencial de abrasão da pele e fornecer um veículo para a introdução de organismos no local.

Estudos epidemiológicos recentes relataram fômites na transmissão de patógenos humanos em ambientes de alta exposição, como hospitais, creches, instituições de longa permanência, instalaçõs esportivas, entre outros. Vários tipos de micro-organismos foram identificados, incluindo rotavírus, rinovírus, Staphylococcus aureus resistente à meticilina (MRSA), Escherichia coli, Pseudomonas 
aeruginosa, Serratia marcescens, (RUTALA et al., 2006; DE JONG et al., 2008; ASHGAR, EL-SAID; 2012; HAILEGIYORGIS, SARHIE, WORKIE, 2018). De acordo com Al-Harbi, Anderson, Elmi (2017), o conhecimento do tipo de micro-organismos existentes em diferentes locais e objetos ajudaria na seleção das medidas de higiene adequadas para suprimir qualquer potencial contaminação cruzada microbiana. Devem ser considerados também outros fatores que afetam a taxa de transferência de bactérias de uma superfície para outra além do tipo de micro-organismo, a origem dos mesmos, o tempo de permanencia após inoculação, a temperatura e o nível de umidade necessários para a sobrevivência ( $D E J O N G$ et al., 2008).

\section{CONCLUSÃO}

De acordo com a metodologia empregada e pelos resultados obtidos pode concluir que não foram detectados alterações nos limiares auditivos na avaliação audiológica convencional na maioria $(91,66 \%)$ dos estudantes universitários e que a audiometria tonal de alta frequência é de suma importância para o diagnóstico precoce da perda auditivo. Ao qualificar, quantificar e correlacionar as colônias de bactérias e fungos encontrados nos plugs de inserção e no conduto auditivo externo, tanto da orelha direita quanto da orelha esquerda pode-se evidenciar que as colônias de fungos e bactérias estão presentes em grandes quantidades e de diversas espécies, sendo Candida albicans, Staphylococcus aureus e Micrococcus spp isolados com maior frequência, tanto nos plugs de inserção como no conduto auditivo externo dos estudantes universitários.

\section{REFERÊNCIAS}

AL-HARBI, M.; ANDERSON, A.; ELMI, A. Evaluation of Microbial Contamination in Frequently Used Fomites in Kuwait. Biodiversity International Journal, v. 1, n. 3, 00013, 2017. http://dx.doi.org/10.15406/bij.2017.01.00013.

ARAÚJO, A. S. Perda auditiva induzida pelo ruído em trabalhadores de metalúrgica. Revista Brasileira de Otorrinolaringologia, v. 68, n. 1, p. 47-52, 2002. http://dx.doi.org/10.1590/S003472992002000100008.

ASHGAR, S.; EL-SAID, H. Pathogenic Bacteria Associated with Different Public Environmental Sites in Mecca City," Open Journal of Medical Microbiology, v. 2, n. 4, p. 133-137, 2012. http://dx.doi.org/10.4236/ojmm.2012.24020 .

BARCELOS, D. D.; DAZZI, N. S. Efeitos do mp3 player na audição. Revista CEFAC, v. 16, n. 3, p. 779-791, 2014.

DE JONG, A. et al. Cross-contamination in the kitchen: effect of hygiene measures. Journal of Applied Microbiology, v. 105, n. 2, p. 615-624, 2008. https://doi.org/ 10.1111/j.1365-2672.2008.03875.x

DOBIE, R. A. Noise induced hearing loss. In: Bailey BJ, editor. Head and neck surgery on cd-rom. 2nd edition, New York, Lippincott-Raven; 1998, 238p.

GONÇALVES, C. L.; DIAS, F. A. M. Achados audiológicos em jovens usuários de fones de ouvido. Revista CEFAC, v. 16, n. 4, p. 1097-110, 2014. 
HAILEGIYORGIS, T. T.; SARHIE, W. D.; WORKIE, H. M. Isolation and antimicrobial drug susceptibility pattern of bacterial pathogens from pediatric patients with otitis media in selected health institutions, Addis Ababa, Ethiopia: a prospective cross-sectional study. BMC Ear, Nose and Throat Disorders, v. 18, p. 8-14, 2018. https://doi.org/10.1186/s12901-018-0056-1

HAILU, D. et al. Pathogenic bacteria profile and antimicrobial susceptibility patterns of ear infection at Bahir Dar Regional Health Research Laboratory Center, Ethiopia. SpringerPlus, v. 5, p. 466-471, 2016. https://doi.org/10.1186/s40064-016-2123-7

HODGETTS, W. E.; RIEGER, J. M.; SZARKO, R. A. The effects of listening environment and earphone style on preferred listening levels of normal hearing adults using an mp3 player. Ear and Hearing. v. 28, n. 3, p. 290-297, 2007.

ILECHUKWU, G. C. et al. Bacterial agents of the discharging middle ear among children seen at the University of Nigeria Teaching Hospital. Enugu. The Pan African Medical Journal, v. 26, p. 87, 2017. https://doi.org/ 10.11604/pamj.2017.26.87.9243

KEPPLER, H. et al. Short-term auditory effects of listening to an MP3 player. Archives of Otolaryngology - Head and Neck Surgery, v. 136, n. 6, p. 538-548, 2010. https://doi.org/ 10.1001/ archoto.2010.84.

KNOBEL, K. A. B.; LIMA, M. C. M. P. Prevenção de perda auditiva induzida por níveis de pressão sonora elevada em crianças e adolescentes. In: Tratado de Audiologia 2 ed.Rio de Janeiro:Guanabara Koogan, 2015; p. 229-234

LAITINEN, H. Factors affecting the use of hearing protectors among classical music players. Noise Health, v. 7, n. 26, p. 21-29, 2005.

LOPES, A. C. et al. Estudo dos limiares de audibilidade de altas frequências em crianças ouvintes com idades entre 7 e 13 anos. Distúrbios da Comunicação, v. 19, n. 2, p. 173-180, 2007.

LOPES, A. C. et al. Perda Auditiva Ocupacional: Audiometria Tonal X Audiometria de Altas Frequências. Arquivos Internacionais de Otorrinolaringologia, v. 13, n. 3, p. 293-299, 2009.

LOPES, A. C.; MELO, A. D. P.; SANTOS, C. C. Estudo dos limiares de audibilidade nas altas frequências em trabalhadores da área odontológica. Arquivos Internacionais de Otorrinolaringologia, v. 16, n. 2, p. 226-231, 2012.

MAZLAN, R. et al. Ear infection and hearing loss amongst headphone users. Malaysian Journal of Medical Sciences, v. 9, n. 2, p. 17-22, 2002

MONDELLI, M. F. C. G.; ARGENTIM, J. P.; ROCHA, A.V. Correlation between speech perception and tinnitus before and after the use of hearing aids. Audiology Communication Research, v. 21, e1649, 2016, Epub, 2016. http://dx.doi.org/10.1590/2317-6431-2015-1649.

MULUYE, D. et al. Bacterial isolates and drug susceptibility patterns of ear discharge from patients with ear infection at Gondar University Hospital, Northwest Ethiopia. BMC Ear, Nose and Throat Disorders, v. 13, n. 10, p. 2-5, 2013. https://doi.org/10.1186/1472-6815-13-10 .

OLIVEIRA, R.C. et al. O impacto do ruído em trabalhadores de Unidades de Suporte Móveis. CoDAS, v. 2, n. 3, p. 215-222, 2015.

OLIVEIRA, M.F.F et al . Fones de ouvido supra-aurais e intra-aurais: um estudo das saídas de intensidade e da audição de seus usuários. Audiology - Communication Research, v. 22, e1783, 2017.

http://dx.doi.org/10.1590/2317-6431-2016-1783 
RUTALA W.A. et al. Bacterial contamination of keyboards: Efficacy and functional impact of disinfectants. Infection Control \& Hospital Epidemiology, v. 27, n. 4, p. 372-377, 2006.

SANT'ANA, N.C. Criação de uma ferramenta hipermídia para promoção da saúde auditiva em jovens usuários de dispositivos sonoros portáteis individuais. Dissertação (Mestrado) - Faculdade de Odontologia de Bauru. Universidade de São Paulo Bauru, 2012. 100p.

SEID, A. et al. Bacterial otitis media in all age group of patients seen at Dessie referral hospital, North East Ethiopia. Egyptian Journal of Ear, Nose, Throat and Allied Sciences, v. 14, p. 73-78, 2013. https://doi.org/10.1016/j.ejenta.2013.02.005

SILVESTRE, R.A. et al. High-frequency profile in adolescente sandits relationship with the use of personal stereo devices. Jornal de Pediatria, v. 92, p. 206-211. 2016. http://dx.doi.org/10.1016/j.jped.2015.07.008

TEIXEIRA, C.S.; KÖRBES, D.; ROSSI, A.G. Ruído e equilíbrio: aplicação da posturografia Dinâmica em indústria gráfica. Revista CEFAC, v. 13, n. 1, p. 92-101, 2011.

WASIHUN, A.G.; ZEMENE, Y. Bacterial profile and antimicrobial susceptibility patterns of otitis media in Ayder teaching and referral Hospital, Mekelle University, Northern Ethiopia. Springerplus, v. 4, p. 701-716, 2015. https://doi.org/10.1186/s40064-0151471-z. eCollection 2015.

WINN, W et al. Koneman's color atlas and textbook of diagnostic microbiology. Lippincott Williams \& Wilkins, Baltimore, Md., USA, 2008, 1760p. 